\title{
Designing the Sliding Mode Controller for the Variable Structure System of Electronically Controlled Diesel Engine \\ Yi Xiaobing
}

Key words: Sliding variable structure controller, Nonlinear system,Engine

\begin{abstract}
Sliding Variable Control has been widely intruduced in industrial fields and achieved good results,because it takes strong robustness without parameters affected and anti-jamming features.The electronically controlled diesel engine is a typical multi-disturbance dynamic and nonlinear system of delay.In order to make the electronically controlled diesel engine to achieve excellent speed stability and adapt to different working environment, this paper introduces a variety of sliding variable structure controller to improve the speed of the electronically controller diesel engine.
\end{abstract}

\section{Sliding Mode Control Theory}

Most of the actual control issues are nonlinear, disturbing and uncertain. Hence, it is necessary to adopt a more theoretically optimized nonlinear feedback and transformation-based control system to achieve sound dynamics and thus obtain satisfactory control results ${ }^{[1]}$.

Provided there is a control system,

$$
\hat{x}=f(x, u, t), x \in R^{n}, u \in R^{m}, t \in R, t \in R
$$

The design of the switching function $s(x), s \in R^{m}$ is needed, and then the control function is solved ${ }^{[2]}$ :

$$
u=\left\{\begin{array}{l}
u^{+}(x), s(x)>0 \\
u^{-}(x), \quad s(x)<0
\end{array} \text {, wherein } u^{+}(x) \neq u^{-}(x)\right.
$$

Being in a sliding mode, meeting the accessibility conditions and remaining stable are the three essential qualities that the sliding mode control shall possess ${ }^{[3]}$.

In the present research, the speed control of electronically controlled diesel engine is in a nonlinear state, which is clearly in line with the form of the formula (3);

$$
\left[\begin{array}{l}
\frac{d}{d t} \zeta_{k}^{i}=\zeta_{k+1}^{i} \quad\left(k=1, \mathrm{~L}, r_{i}-1\right) \\
\frac{d}{d t} \zeta_{r_{i}}^{i}=b_{i}\left(\zeta \zeta+\sum_{j=1}^{m} a_{i j}(\zeta \zeta)_{j}\right.
\end{array}\right.
$$

by conversing (4) equation,

$$
L_{g} h(x)=L_{g} L_{f} h(x)=\mathrm{L}=L_{g} L_{f}^{n-2} h(x)=0
$$


we can then arrive at the conditions under which $h(x)$ is solvable ${ }^{[4]}$.

Provided that $x_{1}=\int_{0}^{t} e d t, x_{2}=e=n_{0}-n$

wherein $n_{0}$ denotes the target speed of the diesel engine, $n$ the actual speed and $e$ the speed deviation between the target speed and the actual speed, then it can be converted into:

$$
\left\{\begin{array}{l}
\hat{x}_{1}=x_{2} \\
\hat{x}_{2}=-d_{2} n^{2}-d_{4} n-d_{6}-\left(d_{1} n^{2}+d_{3} n+d_{5}\right) u
\end{array}\right.
$$

Where, $u=\lambda, n=n_{0}-x_{2}$. With the foregoing transformation conditions, the above equation turns into (7) as below:

$$
\left\{\begin{array}{l}
x_{1}=x_{2} \\
\alpha_{2}=k_{2} x_{2}^{2}+k_{4} x_{2}+k_{6}+\left(k_{1} x_{2}^{2}+k_{3} x_{2}+k_{5}\right) u
\end{array}\right.
$$

Wherein $k_{1}=-d_{1} ; k_{2}=-d_{2} ; k_{3}=2 d_{1} n_{0}+d_{3} ; k_{4}=2 d_{2} n_{0}+d_{4}$;

$$
k_{5}=-d_{1} n_{0}{ }^{2}-d_{3} n_{0}-d_{5} ; k_{6}=-d_{2} n_{0}{ }^{2}-d_{4} n_{0}-d_{6} \text {. }
$$

Thus, the formula (7) can be transformed as follows:

$$
\frac{d x}{d t}=\left[\begin{array}{c}
x_{2} \\
k_{2} x_{2}{ }^{2}+k_{4} x_{2}+k_{6}
\end{array}\right]+\left[\begin{array}{c}
0 \\
k_{1} x_{2}{ }^{2}+k_{3} x_{2}+k_{5}
\end{array}\right] u
$$

$$
a_{f} g(x)=\left[\begin{array}{lc}
0 & 0 \\
0 & 2 k_{1} x_{2}+k_{3}
\end{array}\right]\left[\begin{array}{c}
x_{2} \\
k_{2} x_{2}{ }^{2}+k_{4} x_{2}+k_{6}
\end{array}\right]
$$

Because

$$
\begin{aligned}
& -\left[\begin{array}{cc}
0 & 1 \\
0 & 2 k_{2} x_{2}+k_{4}
\end{array}\right]\left[\begin{array}{c}
0 \\
k_{1} x_{2}^{2}+k_{3} x_{2}+k_{5}
\end{array}\right] \\
& =\left[\begin{array}{c}
-\left(k_{1} x_{2}^{2}+k_{3} x_{2}+k_{5}\right) \\
\left(k_{1} k_{4}-k_{2} k_{3}\right) x_{2}^{2}+2\left(k_{1} k_{6}-k_{2} k_{5}\right) x_{2}+\left(k_{3} k_{6}-k_{4} k_{5}\right)
\end{array}\right]
\end{aligned}
$$

When $x=0$, the rank of the matrix (9) is 2 .

$$
\left[g(x) \quad a d_{f} g(x)\right]=\left[\begin{array}{cc}
0 & -k_{5} \\
k_{5} & \left(k_{3} k_{6}-k_{4} k_{5}\right)
\end{array}\right]
$$

Hence, keep the rank of the matrix condition is satisfied. According to the Involution Distribution Theorem, if the $\left[f_{1}, f_{2}\right]$ of any two vectors distributing in $\Delta$ is still in the distribution, then this distribution is involutory, given one-dimensional distribution is surely involutory. This shows that the involutory condition is met ${ }^{[5]}$.

Thus, the solution of $h(x)$ can be obtained: 


$$
\frac{\partial h}{\partial x} g(x)=0
$$

namely, $\quad\left[\begin{array}{ll}\frac{\partial h}{\partial x_{1}} & \frac{\partial h}{\partial x_{2}}\end{array}\right]\left[\begin{array}{c}0 \\ k_{1} x_{2}{ }^{2}+k_{3} x_{2}+k_{5}\end{array}\right]=0$

(12) thus $h(\mathbf{x})=x_{1}$.

\section{Designing of the controller}

Most of the control systems in reality are nonlinear ones and it is hard to improve them with the existent methods that are put up for linear systems. As for nonlinear control systems ${ }^{[6]}$,

$$
\begin{aligned}
& \hat{x}=f(x, u, t) \\
& x \in R^{n}, u \in R^{m}, t \in R
\end{aligned}
$$

the switching function vector shall be determined

$$
s(x), s \in R^{m}
$$

and the value of the variable structure control shall be obtained ${ }^{[7]}$.

$$
u_{i}(x)= \begin{cases}u_{i}^{+}(x) & s_{i}(x)>0 \\ u_{i}^{-}(x) & s_{i}(x)<0\end{cases}
$$

As for a specific electronically controlled diesel engine, the sliding mode control of speed is as follows ${ }^{[8]}$ :

If the speed control dynamic equation takes the linear switching function

$$
s(\mathbf{x})=c_{1} x_{1}+c_{2} x_{2}+, \mathrm{L},+c_{n-1} x_{n-1}+x_{n}
$$

we can design its controller.

To convert to equivalent control and solve the sliding motion, it is assumed that

$$
\frac{d}{d t} s=\frac{d}{d t} x_{n}+\sum_{i=2}^{n} c_{i-1} \frac{d}{d t} x_{i-1}=\sum_{i=2}^{n} c_{i-1} x_{i}+\alpha(\mathbf{x})+\beta(\mathbf{x}) u=0
$$

Suppose all $\mathbf{x} \in Q \subseteq R^{n}$ ( $Q$ is the point-set of $\mathbf{x}, \quad \beta(\mathbf{x}) \neq 0$, then the equivalent control is:

$$
u_{e q}(\mathbf{x})=-\beta^{-1}(\mathbf{x})\left[\alpha(\mathbf{x})+\sum_{i=2}^{n} c_{i-1} x_{i}\right]
$$

By putting equation (15) and equation (16) together, we can get:

$$
\left[\begin{array}{l}
\frac{d}{d t} x_{n}=-\left(c_{1} x_{2}+c_{2} x_{3}+\mathrm{L}+c_{n-1} x_{n}\right) \\
s(\mathbf{x})=c_{1} x_{1}+c_{2} x_{2}+\mathrm{L}+c_{n-1} x_{n-1}+x_{n}=0
\end{array}\right.
$$

It is equivalent to $s(\mathbf{x})=c_{1} x_{1}+c_{2} x_{2}+\mathrm{L}+c_{n-1} x_{n-1}+x_{n}=0$

which can be also put as: 


$$
x_{1}^{(n-1)}+c_{n-1} x_{1}^{(n-2)}+\mathrm{L}+c_{2} x_{1}^{(1)}+c_{1} x_{1}=0
$$

Make the roots $\lambda_{i}(i=1, \mathrm{~L}, n-1)$ of its characteristic equation

$$
p^{n-1}+c_{n-1} p^{n-2}+\mathrm{L}+c_{2} p+c_{1}=0
$$

on the left half of the complex plane and then select the sliding mode parameters $c_{i}(i=1, \mathrm{~L}, n-1)$,to achieve the stability.

The switching function can be obtained:

$$
s=c x_{1}+x_{2}
$$

The system is switched to the sliding mode, namely,

$$
s=c x_{1}+x_{2}=\aleph_{1}+c x_{1}=0
$$

Its solution is

$$
x_{1}(t)=x_{1}(0) e^{-c t}
$$

Obviously, only when $c>0$, the solution from the above equation is stable; also, with $\frac{d s}{d t}=0$, the equivalent control $u_{e q}(\mathbf{x})$ could be obtained as follows:

$$
\begin{aligned}
& u_{e q}(\mathbf{x})=\frac{-d_{2} n^{2}-d_{4} n-d_{6}+c x_{2}}{d_{1} n^{2}+d_{3} n+d_{5}} \\
& \quad x_{1}=\int_{0}^{t} e d t, x_{2}=e=n_{0}-n
\end{aligned}
$$

Wherein $n_{0}$ denotes the target speed of the diesel engine, $n$ the actual speed, and $e$ the speed deviation of the two speeds, so it can be converted into:

$$
\left\{\begin{array}{l}
\hat{x}_{1}=x_{2} \\
\hat{x}_{2}=-d_{2} n^{2}-d_{4} n-d_{6}-\left(d_{1} n^{2}+d_{3} n+d_{5}\right) u
\end{array}\right.
$$

Wherein, $u=\lambda, n=n_{0}-x_{2}$, is formally equivalent to $\alpha\left(x_{1}, x_{2}\right)+\beta\left(x_{1}, x_{2}\right) u$. By adopting the reaching law approach to solve the systematic sliding mode control, we take the index reaching law as:

$$
\S=-\varepsilon \operatorname{sgn}(s)-k s, \quad \varepsilon, k>0
$$

As such, the structure of the sliding mode controller could be determined as follows: 


$$
\begin{cases}u^{+}=\frac{-d_{2} n^{2}-d_{4} n-d_{6}+c x_{2}+\varepsilon+k s}{d_{1} n^{2}+d_{3} n+d_{5}} & s>0 \\ u^{0}=\frac{-d_{2} n^{2}-d_{4} n-d_{6}+c x_{2}}{d_{1} n^{2}+d_{3} n+d_{5}} & s=0 \\ u^{-}=\frac{-d_{2} n^{2}-d_{4} n-d_{6}+c x_{2}-\varepsilon+k s}{d_{1} n^{2}+d_{3} n+d_{5}} & s<0\end{cases}
$$

\section{Conclusions}

According to sliding model control theory,the paper design a controller to improve the speed.controller. From the model diagram of the diesel engine speed control in Matlab, we can see the inputs were $\mathrm{n}$ and $n_{0}-n$, namely, $x_{2}$. To achieve a better speed control of the diesel engine and conform to its real characteristics, this study set the air-fuel ratio for adjustment. The standard air-fuel ration is set at 17 and whenever it is less than 17, proper combustion is assured. When $c=30, \varepsilon=50$ and $k=0.35$ in the testing, the controller of the model achieved good results and tracked the non-linear speed control, yielding a slight difference between the actual speed and the target speed, with the static error of $0.5 \%$, the instantaneous dynamic error of $2 \%$, and the response speed of 4 seconds. This shows our model can control the speed well.

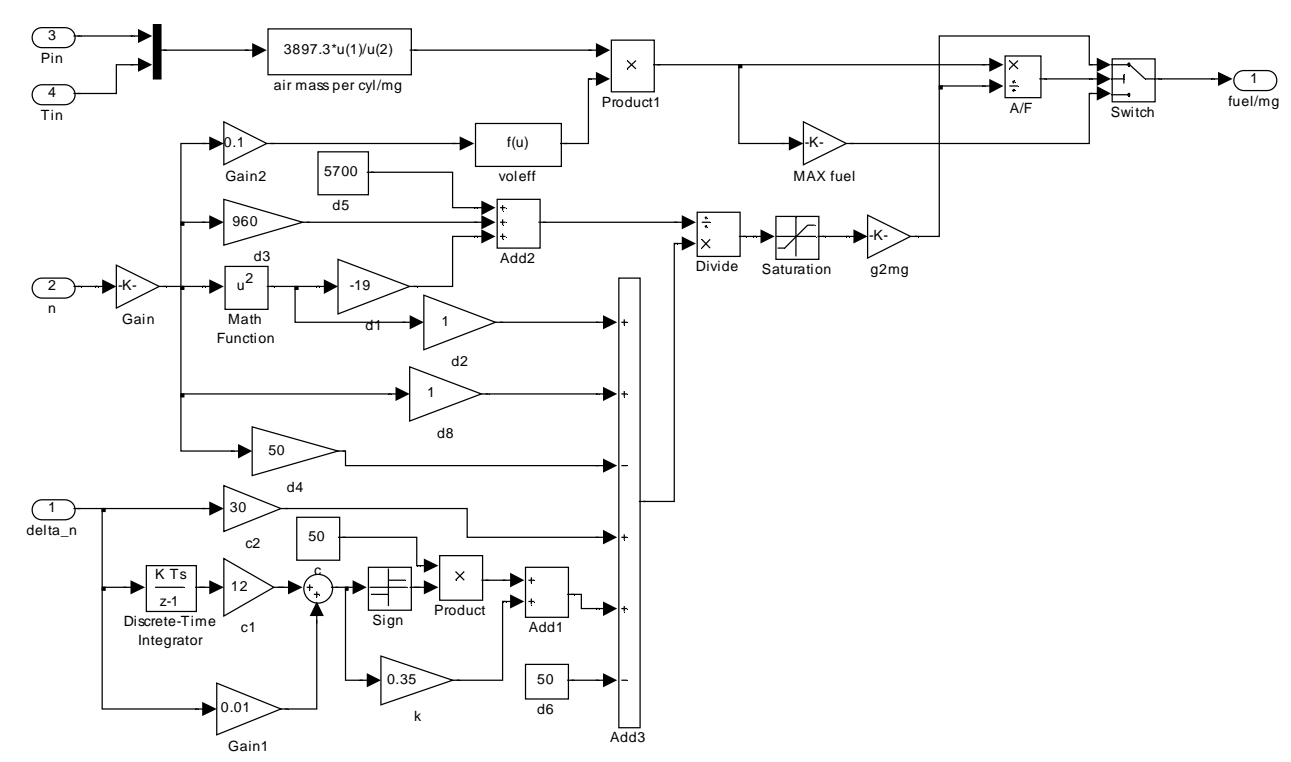

Figure 1 Model of the Sliding Mode Control for Varible Structure System of Electronic Controlled Diesel Engine

By simulation, the result is shown in Figure 2-4. 


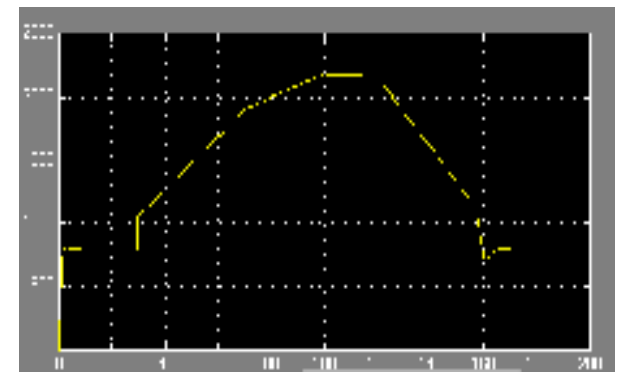

Figure 2 Simulation Diagram of Diesel

Engine Speed Control

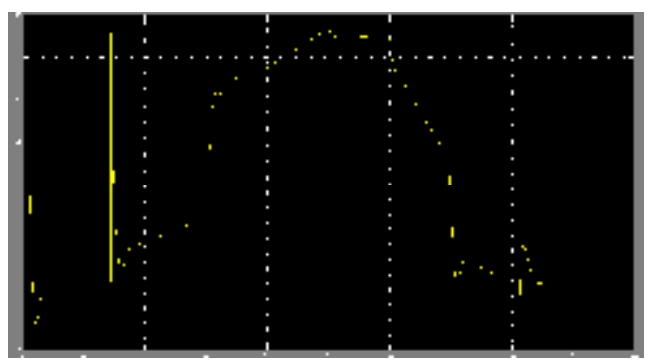

Figure 3 Simulation Diagram of Diesel

Engine Fuel Injection

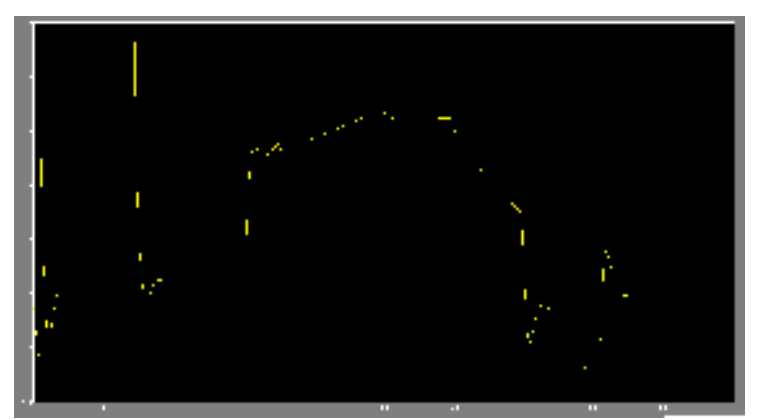

Figure 4 Simulation Diagram of Diesel Engine Torque

[1] Utkin V. I. . Sliding mode and their application in VSSs, Moscow, 1978

[2] Itkin U. . Control systems of variable structure, John wiley \& Sons, 1976

[3] Slotine J J E,Sastry S S. Tracking control of non-lonear systems using sliding surfaces, with application to robot manipulators[J]. Control,1983,38:465-492

[4] Grahaw Wheeler. A sliding mode control for linear systems with improved adaptive laws for the upper bounds on the norm of uncertainties [J]. Automatica,1998,34(2):1657-1661

[5] Brahma, A.; Upadhyay, D. Modeling identification and state estimation of diesel engine torque and NOx dynamics in response to fuel quantity and timing excitations. American Control Conference. 2004

[6] Bartolini G; Ferrara A ;Usai E ;et al . On Multi input Chattering free second order sliding mode control [J ] . IEEE Transactions on Automatic Control , 2000 , 45 (9) : 171121717.

[7] Bartolini G, Ferrara A, Usai E. Chattering avoidance by second order sliding mode control [ J ] . IEEE Transactions on Automatic Control ,1998 , 43 (2) :2412246.

[8] Benedikt Alt;Jan Peter Blath; Ferdinand Svaricek; Matthias Sehultalbers.Control of idle engine Speed and torque reserve with higher order sliding modes[C $\rfloor$.IEEE International Conference on Control Applications, July 8-10,2009. 